\title{
COEFFICIENT ESTIMATES FOR RUSCHEWEYH DERIVATIVES
}

\author{
MASLINA DARUS and AJAB AKBARALLY
}

\author{
Received 5 September 2003
}

We consider functions $f$, analytic in the unit disc and of the normalized form $f(z)=$ $z+\sum_{n=2}^{\infty} a_{n} z^{n}$. For functions $f \in \bar{R}_{\delta}(\beta)$, the class of functions involving the Ruscheweyh derivatives operator, we give sharp upper bounds for the Fekete-Szegö functional $\left|a_{3}-\mu a_{2}^{2}\right|$.

2000 Mathematics Subject Classification: 30C45.

1. Introduction. Let $S$ denote the class of normalized analytic univalent functions $f$ defined by

$$
f(z)=z+\sum_{n=2}^{\infty} a_{n} z^{n}
$$

in the unit disc $D=\{z:|z|<1\}$. Suppose that

$$
\begin{gathered}
S^{*}=\left\{f \in S: \operatorname{Re}\left(\frac{z f^{\prime}(z)}{f(z)}\right)>0, z \in D\right\}, \\
S^{*}(\beta)=\left\{f \in S:\left|\arg \left(\frac{z f^{\prime}(z)}{f(z)}\right)\right|<\frac{\beta \pi}{2}, z \in D\right\}
\end{gathered}
$$

are classes of starlike and strongly starlike functions of order $\beta(0<\beta \leq 1)$, respectively. Note that $S^{*}(\beta) \subset S^{*}$ for $0<\beta<1$ and $S^{*}(1)=S^{*}$ [5]. Kanas [2] introduced the subclass $\bar{R}_{\delta}(\beta)$ of function $f \in S$ as the following.

DEFINITION 1.1. For $\delta \geq 0, \beta \in(0,1]$, a function $f$ normalized by (1.1) belongs to $\bar{R}_{\delta}(\beta)$ if, for $z \in D-\{0\}$ and $D^{\delta} f(z) \neq 0$, the following holds:

$$
\left|\arg \frac{z\left(D^{\delta} f(z)\right)^{\prime}}{D^{\delta} f(z)}\right| \leq \frac{\beta \pi}{2},
$$

where $D^{\delta} f$ denotes the generalized Ruscheweyh derivative which was originally defined as the following.

Definition 1.2 [6]. Let $D^{n} f$ and $f$ be defined by (1.1). Then for $n \in \mathbb{N} \cup\{0\}$,

$$
D^{n} f(z)=\frac{z}{(1-z)^{n+1}} * f(z),
$$

where $*$ denotes the Hadamard product of two analytic functions and $\mathbb{N}$ is a set of natural numbers. 
Later in [1], Al-Amiri generalized the Ruscheweyh derivative $D^{\delta}$ for real numbers $\delta \geq-1$ as a Hadamard product of the functions $f$ and $z /(1-z)^{\delta+1}$.

Note that $\bar{R}_{0}(\beta)=S^{*}(\beta)$ for each $\beta \in(0,1]$ and $\bar{R}_{0}(1)=S^{*}$. In this note, we obtain sharp estimates for $\left|a_{2}\right|,\left|a_{3}\right|$ and the Fekete-Szegö functional for the class $\bar{R}_{\delta}(\beta)$. For the subclass $S^{*}$, sharp upper bounds for the functional $\left|a_{3}-\mu a_{2}^{2}\right|$ have been obtained for all real $\mu[3,4]$.

2. Preliminary results. In proving our results, we will need the following lemmas. However, we first denote $P$ to be the class of analytic functions with positive real part in $D$.

LEMmA 2.1. Let $p \in P$ and let it be of the form $p(z)=1+\sum_{n=1}^{\infty} c_{n} z^{n}$ with $\operatorname{Rep}(z)>0$. Then

(i) $\left|c_{n}\right| \leq 2$ for $n \geq 1$,

(ii) $\left|c_{2}-c_{1}^{2} / 2\right| \leq 2-\left|c_{1}\right|^{2} / 2$.

LEMMA 2.2. Let $\delta \geq 0$ and $\beta \in(0,1]$. If $f \in \bar{R}_{\delta}(\beta)$ and is given by (1.1), then

$$
\begin{gathered}
\left|a_{2}\right| \leq \frac{2 \beta}{\delta+1}, \leq \begin{cases}\frac{2 \beta}{(\delta+2)(\delta+1)} & \text { if } \beta \leq \frac{1}{3} \\
\frac{6 \beta^{2}}{(\delta+2)(\delta+1)} & \text { if } \beta \geq \frac{1}{3} .\end{cases}
\end{gathered}
$$

Proof. Let $F(z)=D^{\delta} f(z)=z+A_{2} z^{2}+A_{3} z^{3}+\cdots$. Since $f \in \bar{R}_{\delta}(\beta)$ and $D^{\delta} f(z) \in$ $S^{*}(\beta)$, then

$$
\frac{z F^{\prime}(z)}{F(z)}=p^{\beta}(z)
$$

and so

$$
\frac{z\left(1+2 A_{2} z+3 A_{3} z^{2}+\cdots\right)}{z+A_{2} z^{2}+A_{3} z^{3}+\cdots}=\left(1+c_{1} z+c_{2} z^{2}+\cdots\right)^{\beta}
$$

which implies that

$$
z+2 A_{2} z^{2}+3 A_{3} z^{3}+\cdots=z+\left(\beta c_{1}+A_{2}\right) z^{2}+\left(\beta c_{2}+\frac{\beta(\beta-1)}{2} c_{1}^{2}+\beta A_{2} c_{1}+A_{3}\right) z^{3}+\cdots
$$

Equating the coefficients, we have

$$
\begin{gathered}
A_{2}=\beta c_{1}, \\
A_{3}=\frac{\beta}{2}\left(c_{2}-\frac{c_{1}^{2}}{2}\right)+\frac{3}{4} \beta^{2} c_{1}^{2} .
\end{gathered}
$$


Now, for $\delta \geq-1, D^{\delta} f$ has the Taylor expansion

$$
D^{\delta} f(z)=z+\sum_{n=2}^{\infty} \frac{\Gamma(n+\delta)}{(n-1) ! \Gamma(1+\delta)} a_{n} z^{n}, \quad z \in D,
$$

where $\Gamma(n+\delta)$ denotes Euler's functions with

$$
\Gamma(n+\delta)=\delta(\delta+1) \cdots(\delta+n-1) \Gamma(\delta) .
$$

Then

$$
z+A_{2} z^{2}+A_{3} z^{3}+\cdots=z+\frac{\Gamma(2+\delta)}{\Gamma(1+\delta)} a_{2} z^{2}+\frac{\Gamma(3+\delta)}{2 \Gamma(1+\delta)} a_{3} z^{3}+\cdots
$$

Equating the coefficients in (2.9), we have

$$
a_{2} \frac{\Gamma(2+\delta)}{\Gamma(1+\delta)}=a_{2}(\delta+1)=A_{2} .
$$

Then, from (2.5), we obtain

$$
a_{2}=\frac{\beta c_{1}}{\delta+1}
$$

It follows that from Lemma 2.1(i)

$$
\left|a_{2}\right| \leq \frac{2 \beta}{\delta+1}
$$

whereas the coefficient of $z^{3}$ in (2.9) is

$$
a_{3} \frac{\Gamma(3+\delta)}{2 \Gamma(1+\delta)}=a_{3} \frac{(\delta+1)(\delta+2)}{2}=A_{3} .
$$

From (2.6), we obtain

$$
a_{3}=\frac{2}{(\delta+1)(\delta+2)}\left[\frac{\beta}{2}\left(c_{2}-\frac{c_{1}^{2}}{2}\right)+\frac{3}{4} \beta^{2} c_{1}^{2}\right] .
$$

It follows from Lemma 2.1(ii) that

$$
\left|a_{3}\right| \leq \frac{2}{(\delta+1)(\delta+2)}\left[\frac{\beta}{2}\left(2-\frac{\left|c_{1}\right|^{2}}{2}\right)+\frac{3}{4} \beta^{2}\left|c_{1}\right|^{2}\right],
$$

that is,

$$
\left|a_{3}\right| \leq \begin{cases}\frac{2 \beta}{(\delta+2)(\delta+1)} & \text { if } \beta \leq \frac{1}{3} \\ \frac{6 \beta^{2}}{(\delta+2)(\delta+1)} & \text { if } \beta \geq \frac{1}{3} .\end{cases}
$$


3. Results. We first consider the functional $\left|a_{3}-\mu a_{2}^{2}\right|$ for complex $\mu$.

THEOREM 3.1. Let $f \in \bar{R}_{\delta}(\beta)$ and $\beta \in(0,1]$. Then for $\mu$ complex,

$$
\left|a_{3}-\mu a_{2}^{2}\right| \leq \frac{2 \beta}{(\delta+1)(\delta+2)} \max \left[1, \frac{|\beta(3(\delta+1)-2 \mu(\delta+2))|}{(\delta+1)}\right] .
$$

For each $\mu$ there is a function in $\bar{R}_{\delta}(\beta)$ such that equality holds.

Proof. From (2.11) and (2.14), we write

$$
\begin{aligned}
a_{3}-\mu a_{2}^{2} & =\frac{2}{(\delta+1)(\delta+2)}\left[\frac{\beta}{2}\left(c_{2}-\frac{c_{1}^{2}}{2}\right)+\frac{3}{4} \beta^{2} c_{1}^{2}\right]-\mu\left(\frac{\beta c_{1}}{\delta+1}\right)^{2}, \\
& =\frac{1}{(\delta+1)(\delta+2)}\left[\beta\left(c_{2}-\frac{c_{1}^{2}}{2}\right)\right]+\frac{\beta^{2}(3(\delta+1)-2 \mu(\delta+2))}{2(\delta+1)^{2}(\delta+2)} c_{1}^{2} .
\end{aligned}
$$

It follows from (3.2) and Lemma 2.1(ii) that

$$
\begin{aligned}
\left|a_{3}-\mu a_{2}^{2}\right| & \leq \frac{\beta}{(\delta+1)(\delta+2)}\left(2-\frac{\left|c_{1}\right|^{2}}{2}\right)+\left|\frac{\beta^{2}(3(\delta+1)-2 \mu(\delta+2))}{2(\delta+1)^{2}(\delta+2)}\right|\left|c_{1}\right|^{2}, \\
& =\frac{2 \beta}{(\delta+1)(\delta+2)}+\frac{\left|\beta^{2}(3(\delta+1)-2 \mu(\delta+2))\right|-\beta(\delta+1)}{2(\delta+1)^{2}(\delta+2)}\left|c_{1}\right|^{2},
\end{aligned}
$$

which on using Lemma 2.1(i), that is, $\left|c_{1}\right| \leq 2$, gives

$$
\left|a_{3}-\mu a_{2}^{2}\right| \leq \begin{cases}\frac{2 \beta}{(\delta+1)(\delta+2)} & \text { if } \kappa(\delta) \leq \beta(\delta+1), \\ \frac{\left|\beta^{2}(6(\delta+1)-4 \mu(\delta+2))\right|}{(\delta+1)^{2}(\delta+2)} & \text { if } \kappa(\delta) \geq \beta(\delta+1),\end{cases}
$$

where $\kappa(\delta)=\left|\beta^{2}(3(\delta+1)-2 \mu(\delta+2))\right|$.

Equality is attained for functions in $\bar{R}_{\delta}(\beta)$ given by

$$
\frac{z\left(D^{\delta} f(z)\right)^{\prime}}{D^{\delta} f(z)}=\left(\frac{1+z^{2}}{1-z^{2}}\right)^{\beta}, \quad \frac{z\left(D^{\delta} f(z)\right)^{\prime}}{D^{\delta} f(z)}=\left(\frac{1+z}{1-z}\right)^{\beta},
$$

respectively.

We next consider the cases where $\mu$ is real and prove the following. 
THEOREM 3.2. Let $f \in \bar{R}_{\delta}(\beta)$ and $\beta \in(0,1]$. Then for $\mu$ real,

$$
\left|a_{3}-\mu a_{2}^{2}\right| \leq \begin{cases}\frac{\beta^{2}(6(\delta+1)-4 \mu(\delta+2))}{(\delta+1)^{2}(\delta+2)} & \text { if } \mu \leq \frac{(6 \beta-2)(\delta+1)}{4 \beta(\delta+2)}, \\ \frac{2 \beta}{(\delta+1)(\delta+2)} & \text { if } \frac{(6 \beta-2)(\delta+1)}{4 \beta(\delta+2)} \leq \mu \leq \frac{(2+6 \beta)(\delta+1)}{4 \beta(\delta+2)}, \\ \frac{\beta^{2}(4 \mu(\delta+2)-6(\delta+1))}{(\delta+1)^{2}(\delta+2)} & \text { if } \mu \geq \frac{(2+6 \beta)(\delta+1)}{4 \beta(\delta+2)} .\end{cases}
$$

For each $\mu$, there is a function in $\bar{R}_{\delta}(\beta)$ such that equality holds.

Proof. Here we consider two cases.

Case (i): $\mu \leq 3(\delta+1) / 2(\delta+2)$.

In this case, (3.2) and Lemma 2.1(ii) give

$$
\begin{aligned}
\left|a_{3}-\mu a_{2}^{2}\right| & \leq \frac{\beta}{(\delta+1)(\delta+2)}\left(2-\frac{\left|c_{1}\right|^{2}}{2}\right)+\frac{\beta^{2}(6(\delta+1)-4 \mu(\delta+2))}{4(\delta+1)^{2}(\delta+2)}\left|c_{1}\right|^{2}, \\
& =\frac{2 \beta}{(\delta+1)(\delta+2)}+\frac{\beta^{2}(6(\delta+1)-4 \mu(\delta+2))-2 \beta(\delta+1)}{4(\delta+1)^{2}(\delta+2)}\left|c_{1}\right|^{2},
\end{aligned}
$$

and so, using the fact that $\left|c_{1}\right| \leq 2$, we obtain

$$
\left|a_{3}-\mu a_{2}^{2}\right| \leq \begin{cases}\frac{\beta^{2}(6(\delta+1)-4 \mu(\delta+2))}{(\delta+1)^{2}(\delta+2)} & \text { if } \mu \leq \frac{(6 \beta-2)(\delta+1)}{4 \beta(\delta+2)} \\ \frac{2 \beta}{(\delta+1)(\delta+2)} & \text { if } \frac{(6 \beta-2)(\delta+1)}{4 \beta(\delta+2)} \leq \mu \leq \frac{3(\delta+1)}{2(\delta+2)}\end{cases}
$$

Equality is attained on choosing $c_{1}=c_{2}=2$ and $c_{1}=0, c_{2}=2$, respectively, in (3.2). Case (ii): $\mu \geq 3(\delta+1) / 2(\delta+2)$.

It follows from (3.2) and Lemma 2.1(ii) that

$$
\begin{aligned}
\left|a_{3}-\mu a_{2}^{2}\right| & \leq \frac{\beta}{(\delta+1)(\delta+2)}\left(2-\frac{\left|c_{1}\right|^{2}}{2}\right)+\frac{\beta^{2}(4 \mu(\delta+2)-6(\delta+1))}{4(\delta+1)^{2}(\delta+2)}\left|c_{1}\right|^{2}, \\
& =\frac{2 \beta}{(\delta+1)(\delta+2)}+\frac{\beta^{2}(4 \mu(\delta+2)-6(\delta+1))-2 \beta(\delta+1)}{4(\delta+1)^{2}(\delta+2)}\left|c_{1}\right|^{2},
\end{aligned}
$$

and so, using the fact that $\left|c_{1}\right| \leq 2$, we obtain

$$
\left|a_{3}-\mu a_{2}^{2}\right| \leq \begin{cases}\frac{2 \beta}{(\delta+1)(\delta+2)} & \text { if } \frac{3(\delta+1)}{2(\delta+2)} \leq \mu \leq \frac{(6 \beta+2)(\delta+1)}{4 \beta(\delta+2)} \\ \frac{\beta^{2}(4 \mu(\delta+2)-6(\delta+1))}{(\delta+1)^{2}(\delta+2)} & \text { if } \mu \leq \frac{(6 \beta+2)(\delta+1)}{4 \beta(\delta+2)}\end{cases}
$$

Equality is attained on choosing $c_{1}=0, c_{2}=2$ and $c_{1}=2 i, c_{2}=-2$, respectively, in (3.2). Thus the proof is complete. 
THEOREM 3.3. Let $f \in \bar{R}_{\delta}(\beta)$ and let it be given by (1.1). Then

$$
\left|a_{3}\right|-\left|a_{2}\right| \leq \frac{2 \beta}{(\delta+1)(\delta+2)} \quad \text { if } \beta \leq \frac{3(\delta+1)}{5 \delta+1} \text {. }
$$

ProOF. Write

$$
\left|a_{3}\right|-\left|a_{2}\right| \leq\left|a_{3}-\frac{2}{3} a_{2}^{2}\right|+\frac{2}{3}\left|a_{2}\right|^{2}-\left|a_{2}\right| .
$$

Then since $(6 \beta-2)(\delta+1) / 4 \beta(\delta+2) \leq 2 / 3$ for $\beta \leq 3(\delta+1) /(5 \delta+1)$, it follows from Theorem 3.2 that

$$
\left|a_{3}\right|-\left|a_{2}\right| \leq \frac{2 \beta}{(\delta+1)(\delta+2)}+\frac{2}{3}\left|a_{2}\right|^{2}-\left|a_{2}\right|=\lambda(x),
$$

where $x=\left|a_{2}\right| \in[0,2 \beta /(\delta+1)]$. Since $\lambda(x)$ attains its maximum value at $x=0$, the theorem follows. This is sharp.

ACKNOWLEDGMENTS. The work presented here was supported by IRPA Grant 09-0202-0080-EA208. The author would like to thank all the referees for their suggestions regarding the contents of the note.

\section{REFERENCES}

[1] H. S. Al-Amiri, Certain generalizations of prestarlike functions, J. Austral. Math. Soc. Ser. A 28 (1979), no. 3, 325-334.

[2] S. Kanas, Class of functions defined by Ruscheweyh derivatives, Bull. Malaysian Math. Soc. 18 (1995), no. 1, 1-8.

[3] F. R. Keogh and E. P. Merkes, A coefficient inequality for certain classes of analytic functions, Proc. Amer. Math. Soc. 20 (1969), 8-12.

[4] W. Ma and D. Minda, A unified treatment of some special classes of univalent functions, Proceedings of the Conference on Complex Analysis (Tianjin, 1992) (Z. Li, F. Y. Ren, L. Yang, and S. Y. Zhang, eds.), Conf. Proc. Lecture Notes Anal., vol. 1, International Press, Massachusetts, 1994, pp. 157-169.

[5] M. Obradović and B. J. Santosh, On certain classes of strongly starlike functions, Taiwanese J. Math. 2 (1998), no. 3, 297-302.

[6] S. Ruscheweyh, New criteria for univalent functions, Proc. Amer. Math. Soc. 49 (1975), 109115.

Maslina Darus: School of Mathematical Sciences, Faculty of Science and Technology, Universiti Kebangsaan Malaysia, 43600 Bangi, Selangor Darul Ehsan, Malaysia

E-mail address: mas 1 ina@pkrisc.cc.ukm.my

Ajab Akbarally: School of Mathematical Sciences, Faculty of Science and Technology, Universiti Kebangsaan Malaysia, 43600 Bangi, Selangor Darul Ehsan, Malaysia 


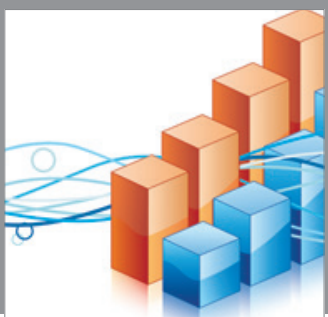

Advances in

Operations Research

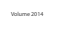

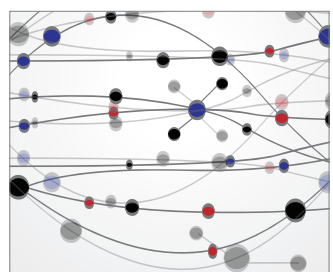

\section{The Scientific} World Journal


International Journal of

Mathematics and

Mathematical

Sciences


Journal of

Applied Mathematics
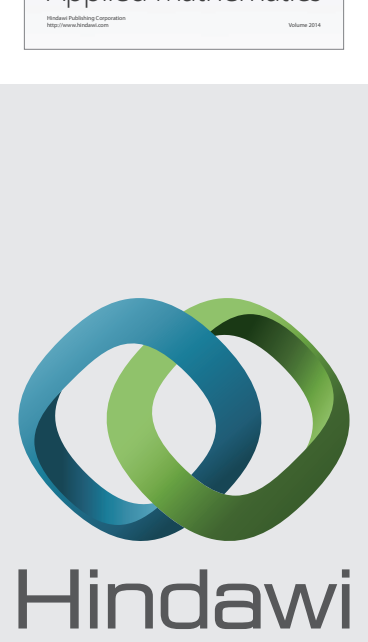

Submit your manuscripts at http://www.hindawi.com
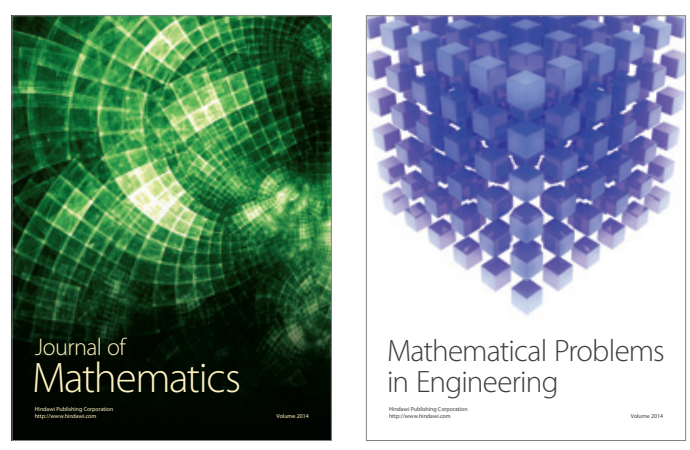

Mathematical Problems in Engineering
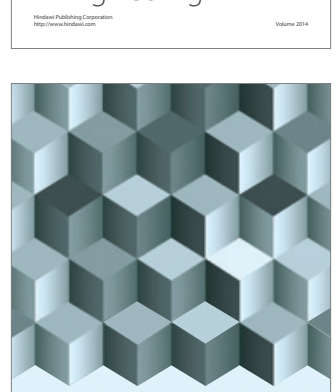

Journal of

Function Spaces
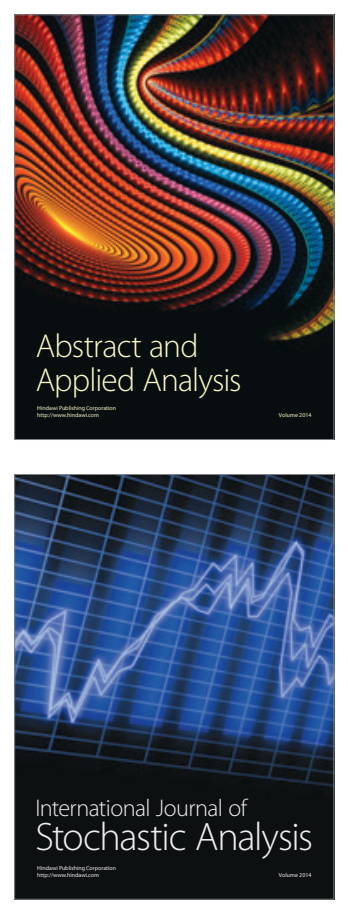

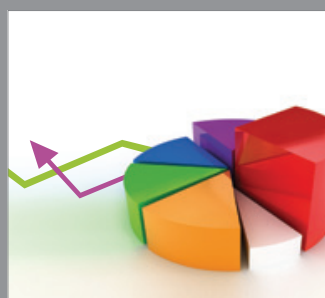

ournal of

Probability and Statistics

Promensencen
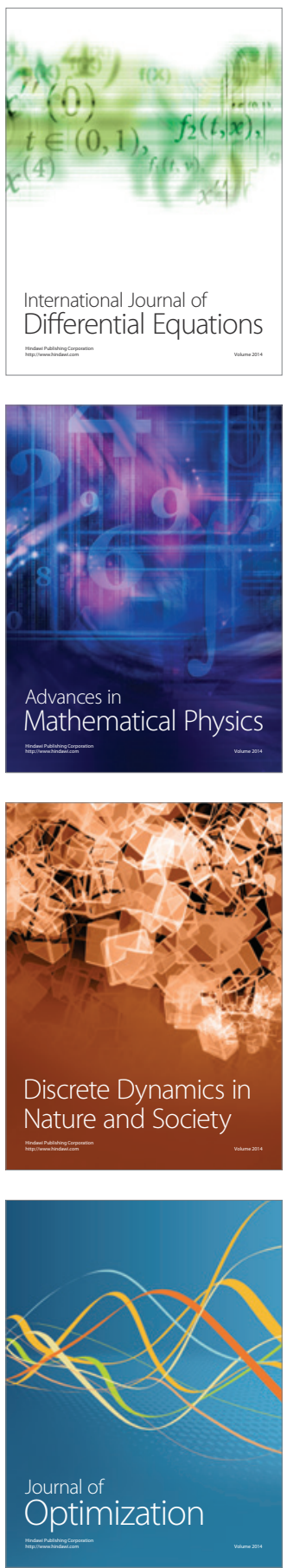